\title{
Definitions and descriptions of networking tools
}

\author{
LAWRENCE H. GINDLER and FREDERICK J. BREMNER \\ Trinity University, San Antonio, Texas
}

\begin{abstract}
This paper provides a comprehensive description of intercomputer communications. The description includes: types of cabling, methods of cabling, network types, hardware employed in networks, software used to operate networks, protocols, file servers, and managers and vendors. A description of the design, development, implementation, and use of a system at a relatively small private institution is also provided.
\end{abstract}

A computer network can be small and simple or large and complex. Small computer networks are often referred to as LANs, which stands for local area networks. In this paper, we will use the word network to mean something larger than a LAN. Principally, this paper discusses the components and the terminology of a campus-wide network (CAWIN). The common thread of a CAWIN is the physical media and interface boxes used to tie it together, known as the backbone (BB). The process of putting together the CAWIN is referred to as networking.

Networking is also both a simple and a complex topic. What the end user sees in a network is simple. The user sees disks, printers, and other devices that are not directly connected to his machine. Behind these connections is a very complex combination of cables, hardware, software, standards, and engineering. The International Standards Organization (ISO) has published a layered description of networks known as the Open Systems Interconnect (OSI) model (Tanenbaum, 1988). This model defines a sevenlayer structure, beginning with the cable at the lowest end and terminating with user applications at the highest end. Unfortunately, no one understands the model. The purpose of this paper is to clarify the OSI model by using a concrete example that is now dynamically in place at Trinity University. We say "dynamically in place" because, like James's stream of consciousness, networking is a continually changing process.

\section{CABLING}

Probably the most mundane aspect of a network is the kind of cable used. However, since this is the part most people associate with a network, it is the place where most descriptions start. The kind of cable does not necessarily dictate the kind of network, but certain cable types are usually associated with a particular network type.

Correspondence should be addressed to F. J. Bremner, Department of Psychology, Trinity University, 715 Stadium Drive, San Antonio, TX 78212. An NSFNET connection for Trinity University: NCR-9021506.

\section{Coaxial Cable}

Coaxial cable (coax) is a center strand of wire covered by a layer of insulation. This layer is then covered by a layer of wire or foil shielding and finally covered by an outer layer of insulation. Coax is usually described by its impedance measured in ohms. The coax used for cable television in your home and for connecting monitors together in the classrooms is 75-ohm cable. This is sometimes called RG59 cable. The coax used for IBM $327 x$ terminals is $92-o h m$ cable. This is often referred to as RG62 cable. The coax used for EtherNet networks (see below) is 50 -ohm cable, referred to as RG58. This cable is used most widely at Trinity University for the networks linking the Sun workstations, the IBM PCs of administrative staff and faculty, and MacIntosh IIs. Although many coaxial cables often look alike, they are not interchangeable. Coax used for terminals cannot be used for cable television or EtherNet.

\section{Twisted-Pair Cable}

Twisted-pair cable is the type used for telephone cords, doorbells, and many low-voltage electrical applications. It consists of "pairs" of wires (each wire with its own insulator) twisted together and covered by an outer layer of insulation. There may also be a shield covering just under the outer layer of insulation. Twisted-pair cable is described as being 1 pair, 2 pair, 25 pair, and so on. The gauge of the wire determines its size. Cable used for Apple LocalTalk, for example, is 1 pair, 22 ga, shielded. Common telephone cord is 2 pair, 22 ga, unshielded. The Trinity University student computer laboratories were originally based on a Corvus system using 1-pair, 22-ga, unshielded twisted pair.

The quality of twisted-pair wire is determined by a level number. Level 1 is recommended for doorbells, Level 2 is used for most telephones, Level 3 is used for data up to $10 \mathrm{MB} / \mathrm{sec}$, and Level 4 is used for data up to $16 \mathrm{MB} / \mathrm{sec}$.

\section{Fiber-Optic Cable}

Fiber-optic cable is a thin strand of glass through which light passes. This light carries a signal in much the same way that the current in the wire cables carries a signal. 


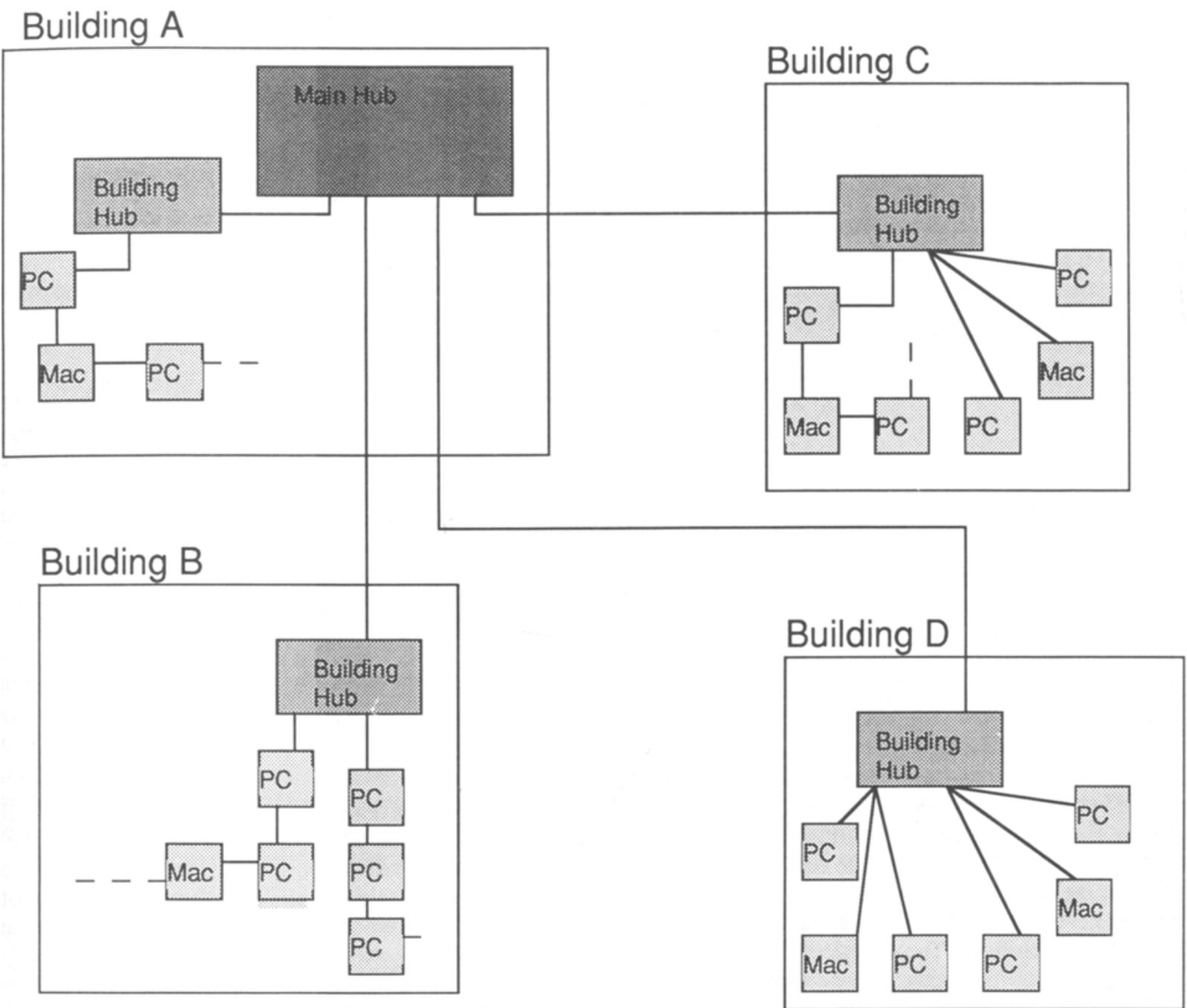

Figure 1. Diagram of CAWIN hardware using hubs, cables, and driver cards.

Because there is no current in fiber cable, it is not affected, for example, by running through wet manholes or near light fixtures. The cable is not expensive, but the connectors used with the cable are. It is difficult to splice if broken. Fiber cable is the cable of choice between buildings and for long distances. It can be used with several different kinds of networks.

\section{Pulling Cable}

Much of the cost of putting a campus BB in place is the labor involved, such as physically pulling cable between buildings. In general, these cables are installed underground between buildings in pipes (often PVC in today's world) called conduits. All campuses have such conduits, but most of them are full of other wiring and most physical plants do not have good documentation on these conduits. Since conduits are always in limited supply, the control of them is always political and sometimes legalistic. For instance, it is possible that AT\&T owns some of the conduits on your campus. While the labor costs of pulling any of the cables described above are essentially the same, the quality of the conduits is not. In particular, fiber-optic cable requires its own conduit because it will not tolerate torquing, stressing, or shearing forces to the extent that wire cables will. At Trinity University, we were fortunate that suitable conduits were available, enabling us to use fiber-optic cable between buildings. We were also fortunate that it was time to put in a new telephone system, so we used a fiber-optic cable with enough strands to service all of our telephone, computer, and audiovisual needs for at least the next 10 years.

\section{HARDWARE}

The connection between the computer or terminal and the network is usually made through some type of inter- 


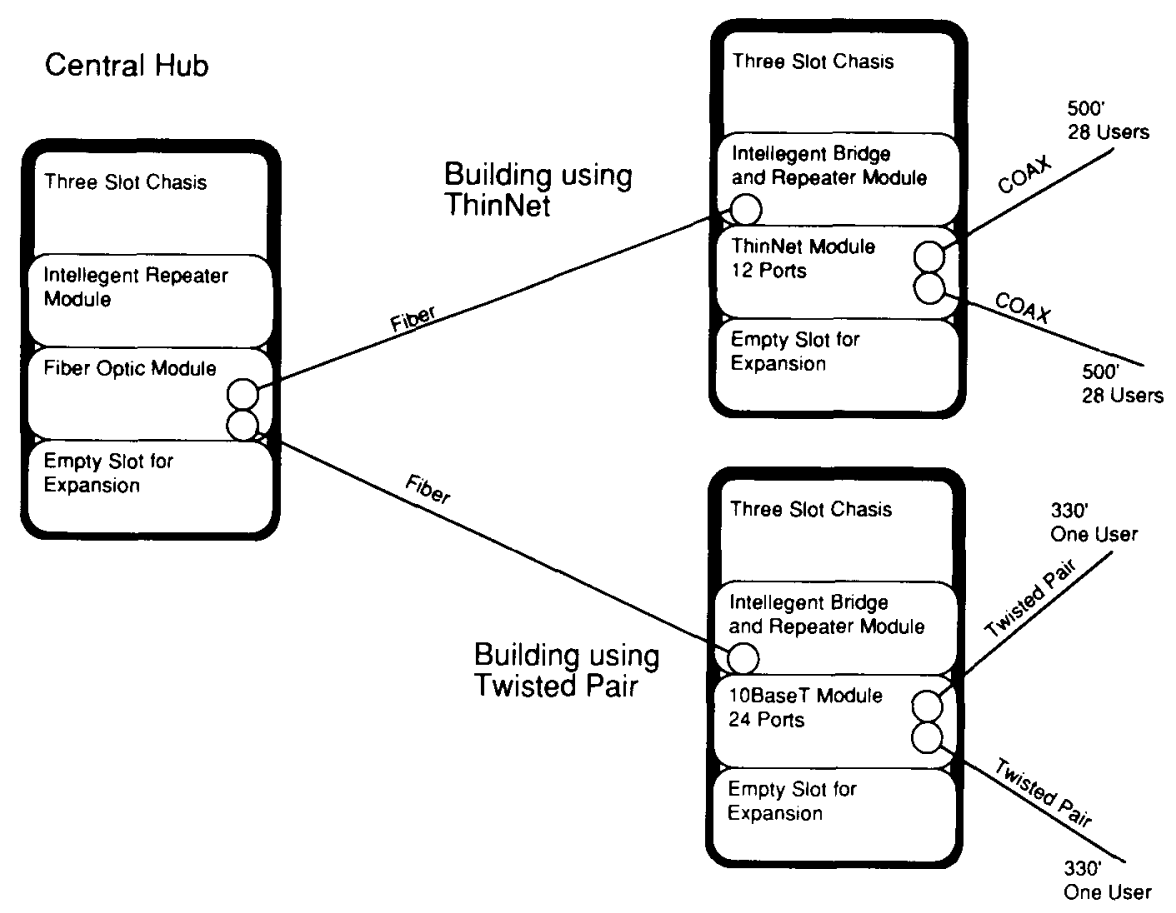

Figure 2. Diagram of CAWIN showing hubs (repeaters and bridges) and cables (fiber optics, coax, and twisted pair).

face card. This card takes the data from the computer and generates the signal over the cable. It also "listens" to the cable for data addressed to it. It is the responsibility of this card to send and receive the data. The card does not determine what to do with data it has received. There are other devices on a CAWIN, called file servers, that aid in information flow. These devices can be dedicated computers or parts of computers on the network. File servers also use software and this will be discussed in the Protocols section below. Regardless of these hardware items (interface cards or file servers), the data are made available to software in the user's computer through a buffer. This software buffer must then decide what to do with the data received. There are a number of different kinds of interface cards and chips available, and they are referred to by their trade names.

\section{EtherNet}

EtherNet was developed in the 1970s as a joint project of Intel, Xerox, and DEC. EtherNet is commonly associated with coax networks, but it works on fiber-optic and twisted-pair cable as well. The primary advantage of EtherNet is that many different machines can connect to an EtherNet network. All machines that are sold to military installations must be capable of connecting to an EtherNet. Therefore, Sun, Apple, IBM, and other computers can be connected to an EtherNet, as well as machines from Xerox, DEC, and Intel. EtherNet cards are available from several manufacturers for IBM PCs and compatibles, Apple Macintosh SEs and Macintosh IIs, most high-level workstations, and many mainframes. However, connection can be made in other ways (see below). The communication between machines on EtherNet is accomplished through

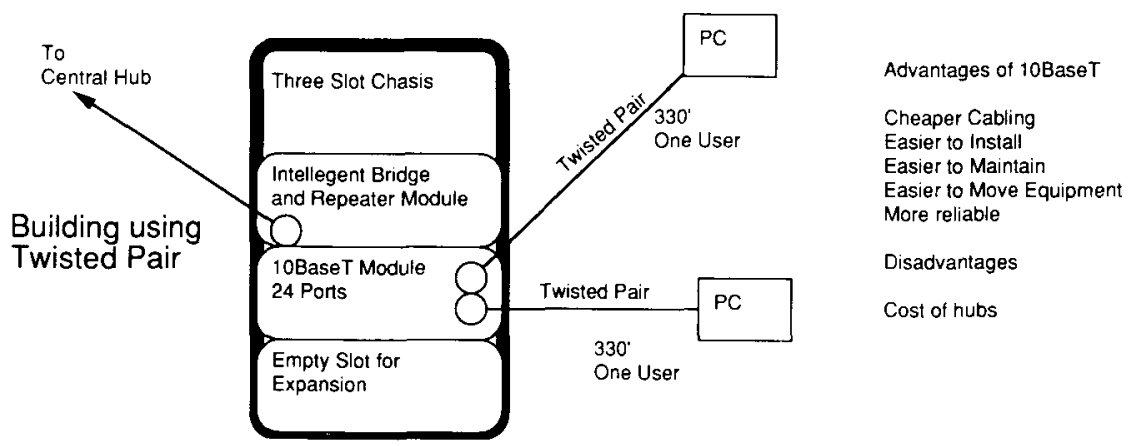

Figure 3. Diagram showing building interface hub with bridge and 24 twisted-pair ports wired in parallel. There are interface cards in each PC. 
what is called Carrier Sense Multiple Access (CSMA) protocols. When a machine has data to send, it listens to the network to see if the line is clear. If the line is clear, then it sends its data. Every machine receives the data and checks the address. However, only the machine to which the data were addressed captures the data. The other machines simply ignore it.

\section{LocalTalk}

Every Apple Macintosh computer has LocalTalk hardware built in. LocalTalk uses one of the serial ports (also used for modems or printers) on the back of the machine and an inexpensive box called a LocalTalk connector. The cable between LocalTalk 22-ga connectors is shielded, 22-ga, twisted pair. The advantage of LocalTalk is that it is inexpensive. The hardware is built into the machines, and the cable is readily available from many sources. The system is somewhat slow and limited to either Macintosh or MS-DOS computers. The basic strategy uses the CSMA protocol described above.

\section{Omninet}

Omninet is a network sold by Corvus Systems. Omninet cards were some of the first network cards available for IBM PCs and clones. Omninet cards are also available for Apple II computers, but we have never used them at Trinity University. Omninet cards are connected to unshielded, twisted-pair cables. Omninet has a major flaw in that one of the pair of wires is used for a send wire and one for a receive wire, with neither being a true ground. Each machine is grounded by the third prong of its standard ac wall plug. Consequently, Omninets cannot be used on computers in separate buildings because the electrical ground level between the buildings can vary by a higher voltage than is used for the signal. The cards can be destroyed by the high-voltage differences.

\section{Token Ring}

IBM has introduced a network technology known as the Token Ring. In the Token Ring, each system must take a turn sending data. A dummy message called the "token" is passed from machine to machine. A machine can send data only when it owns the token. While IBM is betting heavily that Token Ring will be a widely used network, only IBM appears to be firmly behind the Token Ring technology at this time.

\section{Other Cards}

Other network cards of note include Datapoint's ArcNet system, which uses fiber-optic or coaxial cable. AT\&T StarLan uses twisted-pair or fiber-optic cable. Broadband networks by Allen Bradly, Sytek, and Zenith use coaxial or fiber links.

\section{Interface Boxes}

The connection between the cards described above and the backbone is an interface box, which can be of several types: repeaters, bridges, routers, and gateways. These devices are the spines of the backbone and are often used to service all of a department or even an entire building. Repeaters are the simplest of backbone interface boxes; they take signals in, amplify them, and send them out.

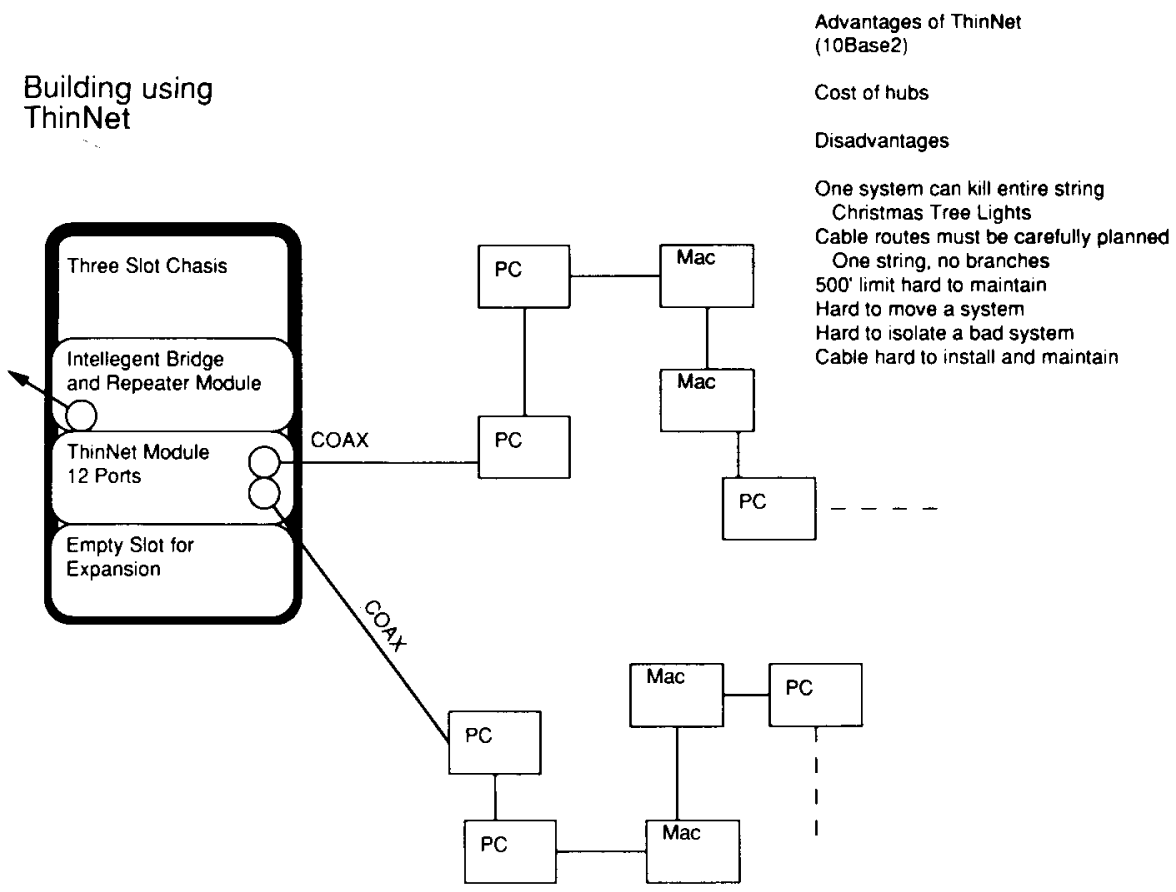

Figure 4. Diagram showing building hub using coax cable wired in parallel but computers wired in serial. There are interface cards in all of the PC machines, but the Macintosh machines have their own interface hardware using one of the serial ports. 


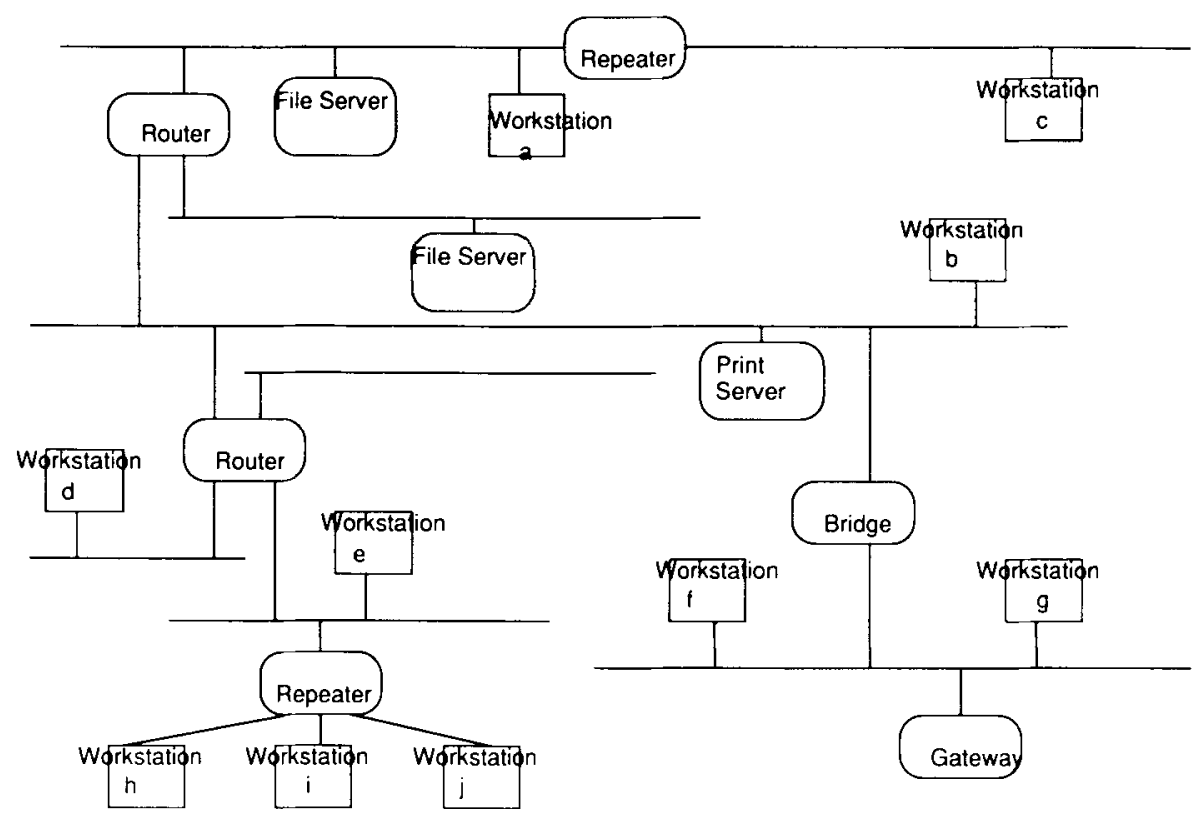

Figure 5. Diagrams illustrating types of hubs (repeaters, routers, bridges, and gateways) and file servers. Workstations a and $c$ are on the same subnetwork logically, even though they are separated by the repeater. Workstation c gets all the traffic to and from Station a. Workstation b, however, is isolated from traffic that is strictly between Stations $f$ and $g$. The bridge between Station $f$ and Station b allows traffic that is addressed to Station $f$ to go to Station $f$ only.

These boxes have from 2 to 24 cable ports that will accept fiber cable, coax, or copper twisted pairs. Any port may be the input port, and the remaining ports handle the output. Input or output port assignments change dynamically. Repeaters are protocol independent.

Bridges are devices that can act like filters. A bridge might be used to tie a department together but prevent the local traffic of that department from being distributed across the entire CAWIN. Bridges are protocol independent, and they learn which addresses are part of a particular network.

Routers, on the other hand, are protocol dependent. Routers designed to route data between Novell workstations will not route data between AppleTalk workstations. While there are some multiprotocol routers that can be programmed to route more than one kind of data packet, they tend to be expensive.

Gateways can convert one protocol to another protocol and are, in that sense, the most sophisticated of interface devices. All of the interface boxes described above represent the spines of the backbone. They are all computers in the broad sense, in that they have central processing units, memory, and input and output lines. They also make decisions (even if they are simple), such as, "If I receive a signal on any of my six lines, then I send it out on my other five lines."

\section{PROTOCOLS}

Each machine on the network must have software that gets the data out of the buffer on the network interface card and makes it available to user programs. Several machines on the same network might use different protocols. If one computer on a network sends information to another machine on a network, the receiving machine can use the data only if the data are in the correct format. Often, the choice of file server software will determine part or all of the network protocol. For example, Novell Netware file servers use a version of EtherNet protocol called IPX. Even though Novell sells no hardware, people say that they have a Novell network. Usually a particular type of network cabling is associated with a particular protocol. However, each kind of cable has more than one type of protocol that can be used, and some protocols are available on a wide range of cable types.

\section{TCP/IP}

Transmission Control Program/Intemet Protocol (TCP/ IP) is commonly used on EtherNets, and it is available on Token Ring and a few other network types. A network purist might argue that only the IP part is the protocol and the TCP part is a collection of user programs; however, for our purposes, we will bundle these two items 
Building With

Department Nets

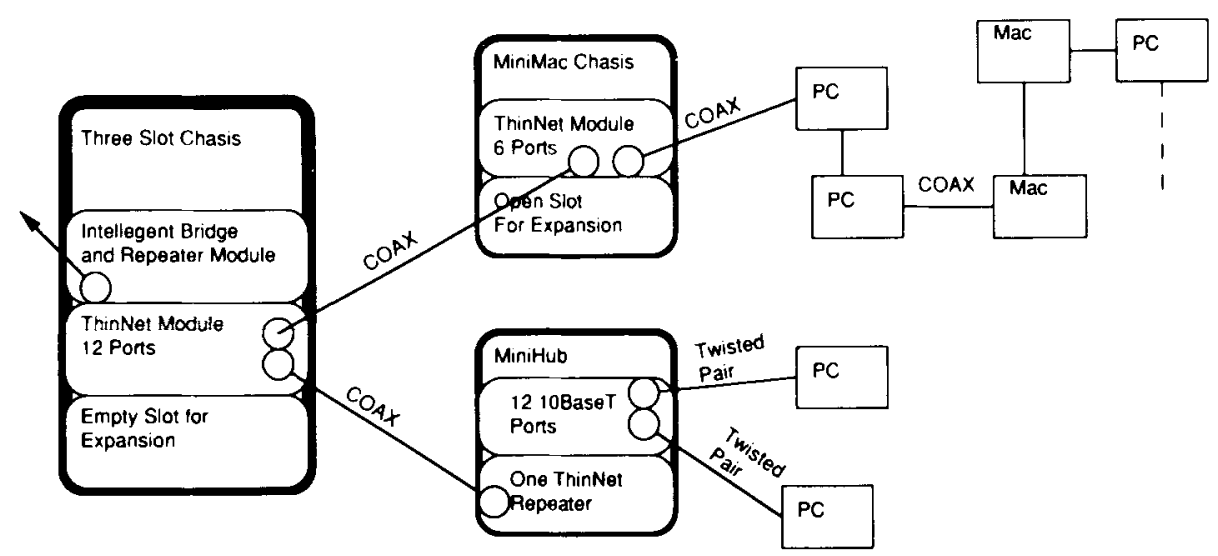

Figure 6. Diagram showing building hub and departmental interface boxes. The minihub is wired in parallel via twisted pairs to PC workstations in the psychology department. The miniMac Chasis is wired in serial to both PC workstations and Mac workstations via coax cable.

together. The TCP/IP is required for military computers so that they can communicate with each other. Because of this, it is available on almost every machine produced. Programs are available through TCP/IP for terminal emulation, file transfer, mail, and messages. Although TCP/IP file servers and disk servers are available for specific machines, they are not the most sophisticated examples of servers for these machines.

\section{OSI}

Open Systems Interconnect (OSI) will replace TCP/IP as the government and military standard over the next several years. At this time, there are few practical applications of OSI, and the deadline for conversion of military systems to OSI is constantly being delayed. The goal of OSI is a network in which the applications, the cable types, the protocols, and so on are independent of each other. This would allow complete interchange of services and information between any vendor's system; however, the goal is not being reached at a very fast pace.

\section{AppleTalk}

Like TCP/IP, AppleTalk is actually a collection of several programs and protocols for network communication. Although AppleTalk was originally developed for LocalTalk networks (both the network and the protocol were called AppleTalk until a few years ago), it is now also used on EtherNets in a variation called EtherTalk and on Token Rings in a variation called TokenTalk. AppleTalk protocols are used by many Apple products by default. All communication between a Macintosh and a LaserWriter is done using the LaserWriter printer driver, an application that uses AppleTalk protocol. ImageWriters on LANs also use AppleTalk. Since AppleTalk is built into the Macintosh operating system, it is easy for devel- opers to write applications that use AppleTalk. Therefore, several software producers have written file servers, print servers, and mail systems for AppleTalk-based networks.

\section{DECNet}

DECNet is used on DEC-Vax, PDP-11, and other machines manufactured by Digital Equipment Corporation. It is a protocol used on EtherNets to connect these systems. Since few DEC systems are used at Trinity, DECNet will not be further described.

\section{XNS}

The Xerox Networking System (XNS) is an EtherNet protocol used by Xerox computers and systems. Although Trinity does not use Xerox computers, a variation of XNS known as IPX is used by Novell's NetWare file servers, print servers, mail programs, and so on. Although XNS was developed and produced for EtherNet, IPX is used by Novell to run its software on virtually every network platform currently sold. Therefore, IPX can be used on Omninets, ArcNets, StarLans, and many others.

\section{NetBios}

IBM, Microsoft, and others are trying to make a PC protocol known as NetBios the standard for PC-based applications. IBM's network software is designed around NetBios. The limitation of NetBios is that it is a PC-only protocol. By using an emulation strategy with their protocols, many network vendors have made it possible to use software written for the NetBios protocol. Emulation of NetBios is available under IPX and TCP/IP.

\section{OS/2 Lan Manager}

Microsoft and IBM have built a series of network servers based on a system known as Lan Manager. Lan 
Manager presently does not compare favorably with other available systems. However, with IBM and Microsoft as its backers, it could become popular.

\section{Proprietary Protocols}

Some protocols were developed for a particular network by the same vendor who builds the network for its own software. DataPoint has its own protocol for its ArcNet, and AT\&T has its own protocol for StarLan. Constellation software on the Omninet uses its own protocol. AppleTalk is actually a proprietary protocol of Apple, but other vendors have adopted it.

\section{Protocols Versus Hardware}

Note that one can run two different protocols on the same hardware at the same time, but machines running different protocols cannot communicate with each other. For example, there may be two PCs on an EtherNet using TCP/IP, transferring files between each other. Two other PCs on the same EtherNet might be running XNS protocols. The two pair of machines will not interfere with each other as long as an XNS machine does not address a data packet to a TCP/IP machine. If this happens, unpredictable results occur. However, the software should prevent this situation from occurring. At the present time, we run both TCP/IP AppleTalk machines and IPX machines on our EtherNet. We also run both IPX machines and Constellation protocols on the OmniNet.

\section{FILE SERVER SOFTWARE}

\section{NetWare}

NetWare by Novell provides software for file servers, print servers, and mail, as well as a foundation for many other applications. Software is available to use with NetWare to connect a network to a mainframe through a gateway, negating the need for each station on the network to have its own connection. Software is available to link stations to shared modems, shared printers, shared CD ROM players, and shared plotters. NetWare will run on almost any cable type, using almost any hardware. The server runs on an AT, PS/ 2 , or other $80 \times 86$ system. A user can also operate the server as a DOS machine; however, if the system crashes, all network users are affected. We currently run NetWare on an EtherNet and an Omninet.

\section{Constellation II}

Constellation II is a disk server and print server software used only on Omninets. It is the software that is shipped with Omninet as a dedicated disk server. Constellation II was one of the first network programs available for IBM PCs and compatibles. It can also run on Apple II computers. It uses its own protocol. The combination of Omninet boards in the PCs and the Constellation II software is what Trinity University students had commonly referred to as "Corvus." Nevertheless, all of our machines with Corvus hardware now run Novell Netware, even though the hardware was not changed.

\section{AppleShare}

AppleShare is Apple's own file server software for AppleTalk-based networks. It will run on either EtherNet or LocalTalk networks and, now, on Token Ring networks. The AppleShare server machine must be only a server. It cannot be used by a Macintosh user while it is functioning as the server. Because AppleShare is a "true" file server, software that is not network compatible does not always function properly on the server. This can be a problem with older applications, but Version 3.0 has solved many of the problems with applications software. Version 3.0 of AppleShare has many features not included with the previous versions, including the ability to run on a machine used both as a server and as a workstation. Version 7.0 of the Macintosh operating system also allows every user to be a "mini AppleShare" file server for up to 10 users.

\section{TOPS}

Tops, from Sitka Corporation, is a set of programs that allow PCs, Macintoshes, and Suns on an EtherNet- or LocalTalk-based network to share files with each other. Unlike AppleShare and NetWare, every machine on the network may make files available to every other machine on the network. This allows any user to make a file available to any other user by "publishing" the file on the network. Tops is the software of choice for an office using Macintoshes and PCs with hard disks on the same small network. However, because each computer on the network is both a file server and a workstation, it is unsuitable for a student laboratory environment. It is also unsuitable for connecting a large number of workstations to a CAWIN.

\section{NFS}

Network File System (NFS) is a file server scheme originally developed for UNIX-based systems using IP protocols. The premise behind NSF is that every system could make its file system available to every other system in a format that could be presented to the user as a local file system. In other words, over this network, the folders on a Macintosh could look like subdirectories to a PC. Since each machine could make its file system available to the network in a common format, the client machine needs only to convert the "standard" file system to its own local system and to make its own local system look to the network like the "standard" system.

\section{Telnet, SMTP, FTP, and TFTP}

TCP/IP protocol allows the use of several standard programs for terminal emulation, mail handling, and file transfer. Telnet is the terminal emulation program. Simple Mail Transfer Protocol (SMTP) is a mail transfer agent. It is not an end user program, but a part of TCP/IP that routes mail to the proper destination. File Transfer Program (FTP) is for large files that include extensive error checking on the transfer. Trivial File Transfer Program (TFTP) is used for smaller files and does not do extensive error check- 


\section{NSFNet/InterNet}

National Science Foundation Network

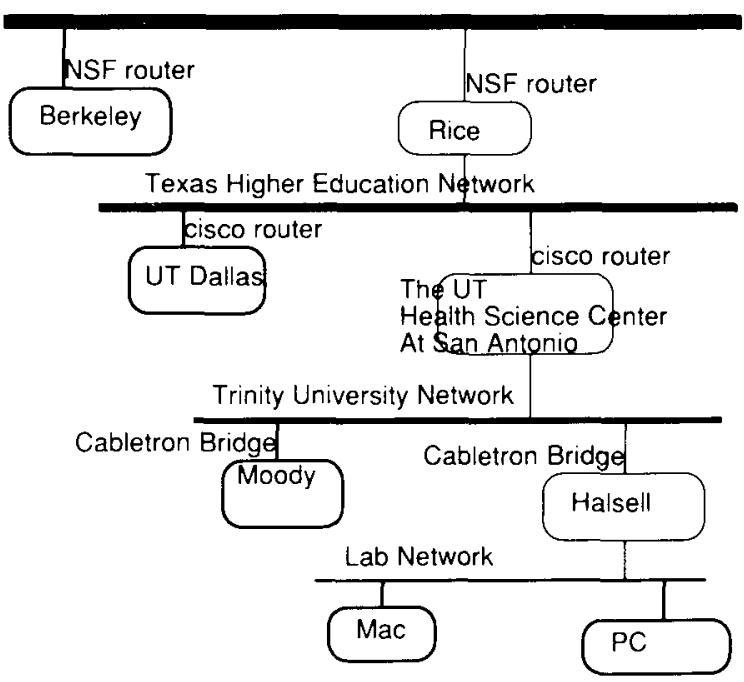

Figure 7. Diagram showing connection of individual computers to CAWIN and CAWIN to National Science Foundation Network.

ing. Finally, TFTP is used for such tasks as remote booting of diskless workstations.

\section{VENDORS}

Cable, hardware, and software can be purchased from a number of vendors. Proprietary items must, of course, be purchased from the vendor who developed each item. Standard items are available from many sources. Some items are licensed to several manufacturers even though the patent or copyright is owned by a single manufacturer.

Price competition is fierce. When certain generic items are needed, a few calls to the mail order companies listed in any of the computer trade magazines can often result in large savings.

\section{Cable}

Cable is commonly purchased from a large distributor, such as Anixter. Name-brand cable will usually be higher priced, but it can be trusted to be properly labeled as to its type and will usually conform to the specifications published for a particular cable type.

Cable that does not meet the electrical specifications for a particular network type can cause very strange network errors. Often a network that "works sometimes and sometimes does not" is the result of nonconforming cable. In addition to electrical characteristics, fire codes often dictate the acceptable outer coverings for the cable. For example, PVC cable, which gives off toxic gases when it burns, is not allowed in ceilings that are used as return air plenums.

\section{Interface Boards}

Competition among makers of interface cards is helping to drastically decrease the cost of adding a workstation to a network. Most systems can now be connected to an EtherNet for under $\$ 200$. Major manufacturers of EtherNet cards include 3-Com, Western Digital, Asante, Cabletron, and others. IBM and Apple also market their own EtherNet cards, but these tend to be higher priced than other, often better cards.

A situation to avoid is having cards from many different makers in the same network. Although this is workable, support for many different kinds of cards can be trying. Each manufacturer's cards have their own drivers and their own idiosyncracies. Limiting the number of different cards makes it much easier to find problems and provide quick solutions.

\section{CONCLUSION}

This paper is not intended to cover every kind of possible network. Its purpose is to introduce the systems and, especially, the terminology that is used when discussing networks, with special emphasis on the systems we are using or trying to use at Trinity University. An end user of network applications needs only to know that his machine extends to the entire network. Users should not have to change their ways of handling tasks to accommodate the network. They should only see the new horizons available when they communicate with others.

\section{REFERENCES}

Tanenbaum, A. S. (1988). Computer networks. Englewood Cliffs, NJ: Prentice-Hall. 